Dynamical Syst ens Anal ysi s of Arterial Bl ood Pressure Si gnal s i $n$ Rel at i on to Heart Rate Fl uct uat i ons in Chi ck Enbryos

\begin{tabular}{|l|l|}
\hline $\begin{array}{l}\text { その他 (別言語等) } \\
\text { のタイトル }\end{array}$ & $\begin{array}{l}\text { ニワトリ胚の心拍ゆらぎに関連した胚動脈圧信号の } \\
\text { カ学系理論による解析 }\end{array}$ \\
\hline 著者 & $\begin{array}{l}\text { AK YANA Ryui chi , NAGASH NA Tonmmasa, TAZAWA } \\
\text { H r oshi }\end{array}$ \\
\hline $\begin{array}{l}\text { j our nal or } \\
\text { publ i cat i on ti tl e }\end{array}$ & Menmi rs of the Mur or an I nst i t ut e of Technol ogy \\
\hline vol une & 49 \\
\hline page r ange & $73-79$ \\
\hline year & 1999 - 11- 30 \\
\hline URL & ht t p: //hdl . handl e. net /10258/155 \\
\hline
\end{tabular}




\title{
Dynamical Systems Analysis of Arterial Blood Pressure Signals in Relation to Heart Rate Fluctuations in Chick Embryos
}

\author{
Ryuichi AKIYAMA*, Tomomasa NAGASHIMA** and Hiroshi TAZAWA** \\ (Accepted 31 August 1999)
}

\begin{abstract}
We attempted a new approach besed on a modern dynamical system theory to reconstruct the arterial blood pressure signals in relation to heart rate fluetuations of developing chick embryos. The dynemical systems approach in general is to model a phenomenon that is presented by a single time series record and approximate the dynamical property (e.g., heart rate fluctuations) of a aystem based only on information contained in a single-variable (erterial blood pressure) of the system. The time-series data of the arterial blood pressure was reconstructed in 3-dimensional space to draw characteristic orbits. Since the reconstzucted orbits of the blood pressure should retain information contained in the pressure signals, we attempted to derive instantaneous heart rate (IHR) from the roconstructed orbits. The derived IfR presenting fiR fluctuations coincided well with the IFR obtained conventionally from the peak-to-peak time intervals of the maximum blood pressure. Movements of the reconstrueted orbits of the arterial blood pressure in 3-dimensional space reflected $\mathrm{HR}$ fluctuations (i.e., transient decelerations and accelerations).
\end{abstract}

Keywords: arterial blood pressure, chick embryo, dynamical systems approach, heart rate fluctuations, instartaneous heart rata, orbit reconstruction, reconstructed orbit, 3-dimensional space

\section{INTRODUCTION}

Incubated avian eggs have been tused as an experimental model for investigation of developmental physiology ${ }^{(3,23)}$. Physiological functions of embryos developing inside an eggshell are completely independent of maternal functions and some physiological variables can easily be measured while maintaining adequate gas exchange through the eggshell. Among those variables, embryonic heart rate of chickens (HR) is determined throughout nearly the whole period of incubation by detecting the cardiogenic signals with various invasive and noninvasive methods $^{(1,2,4,6,8-16,21,22,24)}$. Catheterization of the allantoic artery, which is made by taking advantage of the eggshell, makes it possible to measure the blood pressure during the last half of incubation prior to pipping ${ }^{(19-20,23,26)}$. Allantoic arterial blood pressure was recently used to determine instantaneous heart rate (IHR) of developing chick embryos ${ }^{(7)}$. Blood

Division of Pediatric Cardiology, University of Kentucky;

"Department of Computer sciences and systems

Enginearing:

Wepartment of Electrical and Electronic Engineering was determined from individual peak-to-peak pressure is a simple monophasic wave and IHR intervals of the wave. Various patterns of HR fluctuations were found in late embryos.

From a viewpoint of systems information engineering, the blood pressure wave composed of low-frequency signals and the derived IHR containing HR fluctuations seem to be a favorable biological model for analysis using modern dynamical systems theory (deterministic approach)(5). The dynamical systems approach is to model irregularly time-varying phenomena (time-series data) and approximate the dynamical property of a system based only on information contained in a single variable of the system ${ }^{(17)}$. Although the theory of the dynamical systems approach has been tested in many artificial system models, it has seldom been applied to biological phenomena. Thus in relation to the symposium on cardiac rhythms in animals, we attempted to apply the dynamical systems approach to reconstruct the arterial blood pressure signals as an orbit in a high dimensional space and derive IHR from reconstructed orbits. In the present attempt, the single variable that is used in the dynamical systems approach is arterial blood pressure and the dynamical properties that are derived are the fluctuations of IHR. 


\section{MATERIALS AND METHODS}

\subsection{Arterial blood pressure signals}

In a previous study, the arterial blood pressure of the allantoic circulation was measured to derive IHR and investigate development of HR fluctuations in chick embryos ("). A catheter comprised of a hypodermic needle and polyethylene tube was implanted in the allantoic artery through a small opening in the eggshell. The catheter was fixed onto the eggshell with clay and the opening was closed with vinyl tape and epoxy glue. A conventional strain gauge manometer was connected to the catheter, and the pressure signals were amplified and filtered by a polygraph amplifier to match the input signal level of an analog-to-digital converter. The pressure signals were sampled at $100 \mathrm{~Hz}$, recorded on a microcomputer, and restored by the following sinc function ${ }^{(2)}$ :

$$
\mathrm{BP}(\mathrm{t})=\sum_{j=-\infty}^{\infty} \mathrm{BP}(\mathrm{j} \cdot \mathrm{I}) \cdot \frac{\sin \left[2 \pi f_{N}(\mathrm{t}-\mathrm{j} \cdot \mathrm{I})\right]}{2 \pi f_{k}(\mathrm{t}-\mathrm{j} \cdot \mathrm{I})}
$$

where $I$ is the sampling interval (i.e., $10 \mathrm{~ms}$ ), and $f_{k}$ is Nyquist's reflection frequency (50 (ifz).

Wave restoration by eq. 1 using 401 sampling points ( $i . e ., j=200$ ) was examined to correspond to a signal sampled at $8,000 \mathrm{~Hz}$ (sampling time interval $=125 \mu \mathrm{sec}$ ), ensuring calculation of IHR with an error in accuracy of less than 1 beat/min. After restoration of the systolic pressure wave, the maximum point was found in the restored wave, the time interval between the two adjacent maxima (.t in sec) was determined, and IHR was calculated by $I H R=60 / . t$ (beats $/$ min, bpm). In the present study, we used the arterial blood pressure signals that were measured previously as above. Prior to use of the previously measured pressure signals, we examined algain the accuracy of the pressure signals, which were sampled at $50 \mathrm{~Hz}$ (instead of $100 \mathrm{~Hz}$ ) and restored by eq. 1 with 401 sampling points. In the test experiment, the catheter was implanted in the allantoic artery of an 18-day-old embryo as mentioned above and the arterial pressure was sampled at a frequency of $8,000 \mathrm{~Hz}$ instead of $50 \mathrm{~Hz}$. IHR was calculated from the time intervals between two consecutive peaks of maximum pressure (referred to as $\mathrm{HR}_{\mathrm{g} 000}$ ). Then, single values from every 160 sampling points (which corresponded to pressure signal sampled at $50 \mathrm{kz}$ ) were extracted, and the pressure wave was restored by eq. 1 with 401 sampling points from the extracted values. IHR was calculated for restored pressure waves in the same manner (referred to as $\mathrm{HR}_{\text {rasto }}$ ). It was found that $\mathrm{HR}_{\text {rosto }}$ was consistent with $\mathrm{HR}_{\mathrm{gooo}}$, ensuring the accuracy of the pressure signals that were previously measured at a sampling Irequency of
$100 \mathrm{~Hz}$ and restored by the sinc function.

\subsection{Orbit reconstruction}

In the dynamical systems approach, an orbit (attractor) of a single variable (i.e., blood pressure in the present study) is reconstructed (embedded) in a high dimensional (i.e., m-dimensional) space from the timeseries data of the variable. The embedding dimension, $m$, should be no less than 3 for irregularly time-varying signals. In order to present a pattern of the orbit as a visible figure on a computer screen, we reconstructed the orbit in 3-dimensional space. For time-series data of a given blood pressure, $B P(t)(0<t<T ; T=o n e$ cardiac period), we assumed a suitable time delay $\tau$, which is discretional and determined empirically, . then took two more values, $B P(t+\tau)$ and $B T(t+2 \tau)$. These three values of blood pressure at time $t$ were then put on individual axes of a 3-dimensional rectangular-coordinate, giving a vector with coordinates. An orbit of the vector was drawn by varying time $t$ by $125 \mu \mathrm{sec}$ during one cardiac period with coordinates, thus giving a reconstructed orbit of blood pressure during the T-period. The 3-dimensional orbits were drawn consecutively for time-series data of the blood pressure during $n T$ period where $n$ is discretional real number. The presentation of orbit patterns was made only for a part of the pulse pressure (i,e., during nT period), and the position of 3 -dimensional rectangularcoordinate axes was arbitrarily drawn to present differences in orbit size between young and late embryos, and changes in orbits during the heart beat of individual embryos.

\subsection{Derivation of IHR from reconstructed orbits The reconstructed orbit is another} presentation of blood pressure and must include information contained in the time-series signal of blood pressure; that is, IHR also should be included. Thus we attempted to derive IHR from the reconstructed orbits. If the time delay $\tau$ is considered adequately; empirically a value within one-tenth of the cardiac period $T$, a vector comprising of $[B P(t), B P(t+\tau), B P(t+2 \tau)]$ rotates in the $3-$ dimensional space during one cardiac period and forms an open or closed orbit. When the orbit is not closed, it forms a thin sheet-like configuration during $\mathrm{nT}$ period. Suppose a plane crosses at right angles to the sheetlike orbits at an arbitrary position in space. Then the time interval between points of the orbits which cross the plane in sequence is determined successively (i.e., .t in sec), and the HR is calculated by $H R=60 / . t$ in bpm and plotted on the computer monitor sequentially.

\section{RESULTS}

Figure 1 shows the arterial blood pressure of a 12-day-old embryo (panel A) and its reconstructed orbits (strange attractor) 
drawn in 3-dimensional space (panel B). Blood pressure is presented for $30 \mathrm{~min}$, and pressure
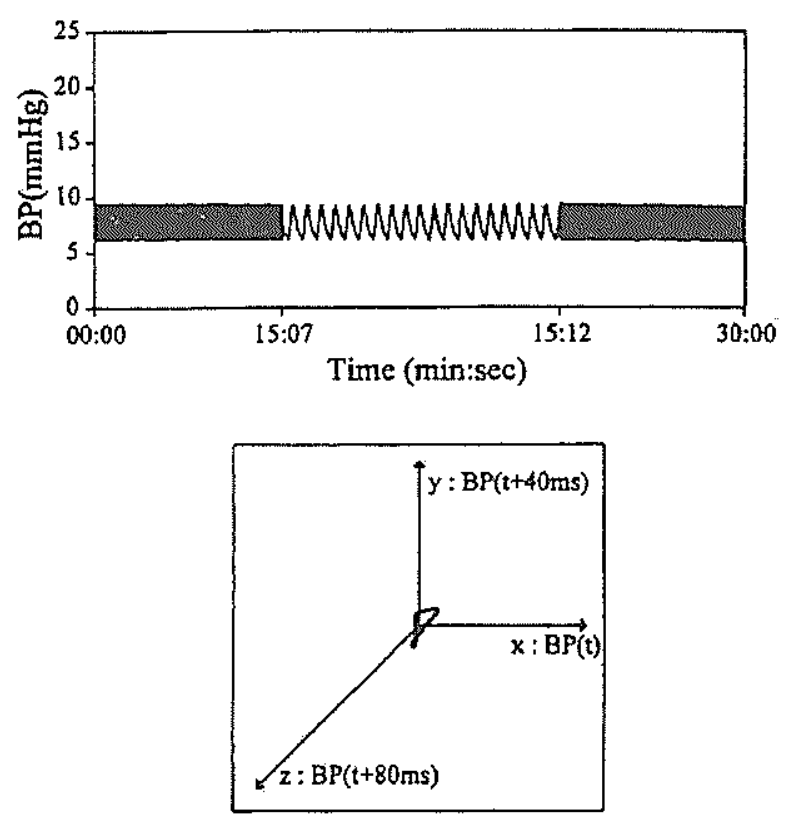

Fig. 1. Time-series record of the arterial blood pressure (top panel) and 18 orbits reconstructed in the 3-dimensional space (bottom panel). The embryo was 12-day-old.
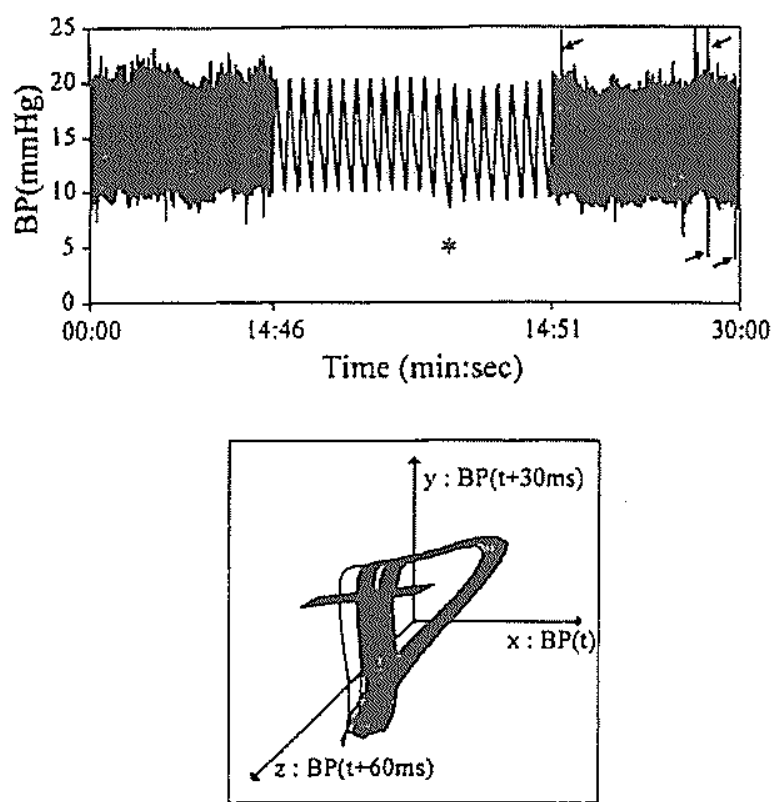

Fig. 2, The arterial blood pressure (top panel) and reconstructed orbits (bottom panel) in an 18day-old embryo. For comparison between Figs. 1 and 2 , the scales for the blood pressure record and reconstructed orbits are identical in both figures.

waves during a $5-$ sec period are shown in the middle of the recording at a faster chart speed (panel A). In young embryos, the blood pressure was low compared with late embryos and formed simple monophasic waves. A single orbit was reconstructed from a blood pressure wave of one-cardiac period, and panel $B$ presents 18 reconstructed orbits which correspond to the pulse pressure waves presented in the middle of panel A. The time delay $(\tau)$ of 40 msec was taken arbitrarily so that the orbits could make characteristic figures.

Figure 2 shows another example of the arterial blood pressure of an 18-day-old embryo (panel $A$ ) and its reconstructed orbits (panel B). Arterial blood pressure increased with embryonic development, but the waves were still monophasic (panel A). As embryos grew, their activities increased and induced artefacts in the pressure signals; i.e., abruptly recorded spikes marked by an arrow. Beside these artefacts, the blood pressure signals fluctuated as shown throughout and in the middle of the recording. The reconstructed orbits were drawn by taking arbitrarily $\tau$ of $30 \mathrm{msec}$ (panel B) for 19 pressure waves during the 5-sec period (14:46-14:51 in panel $A$ ). The orbits moved towards the left along the $x$-axis with heart beats and reached the left most position at the 12th beat, which lowered the minimum pressure (as shown by an asterisk in about the midale of recording in panel A) and thereafter orbits moved towards the right again.

Because a single orbit is reconstructed from a pulse pressure wave during one cardiac period, duration of the cardiac period is known by cutting the orbits by an arbitrary plane (as shown by a thick line in panel B of Fig. 2) and measuring the time interval between points on the orbit crossing the plane successively. Then, the IHR in bpm is calculated from the time interval. Figure 3 presents the same arterial blood pressure as shown in Fig. 2 (panel A), IHR calculated conventionally from peak-topeak time intervals of the blood pressure (panel B), and IHR derived from the reconstructed orbits as mentioned above (panel c) and 18 reconstructed orbits corresponding to a run of heart beats with a stable rate (panel D), decelerated rate (panel $E$ ) and accelerated rate (panel F), respectively. The IHR derived from the reconstructed orbits of the arterial pressure (panel c) concides well with the IHR obtained by conventional calculation from the time interval of the maximum blood pressure (panel B). The reconstructed orbits for 18 cardiac periods during HR deceleration and acceleration present different changes in patterns and movements between them. When the HR is stable (marked by D in panel $C$, and panel D), the reconstructed orbits of the arterial pressure form a narrow band. When the HR decelerates, the orbits move leftwards along the x-axis, reach the left most position corresponding to the minimum of HR and then move towards the original position obtained for stable HR (i.e., towards the right) (marked by $E$ in panel $C$, and panel E). Contrarily, the 
reconstructed orbits move towards the right along the $x$-axis as the HR accelerates (marked by $F$ in panel $C$, and panel $F$ ).

Figure 4 shows the arterial blood pressure recorded from a 20-day-old embryo (upper tracing in panel $A$ ), the IHR calculated from the time interval of the maximum blood pressure (lower tracing in panel $A$ ) and the reconstructed orbits (panels B, C and D) of blood pressure signals for the period indicated by the letters $B, C$ and $D$ in panel $A$. During a 33-sec recording, a single-beat acceleration and deceleration (B) and then an accelerated HR with single-beat deceleration (D) occurred. The reconstructed orbits present characteristic figures for these HR fluctuations. For 18 pressure signals, the reconstructed orbits present a similar pattern corresponding to stable $\mathrm{HR}$, resulting in narrow band (maxked by $C$ in panel. $A$, and panel $C$ ). The reconstructed orbits of 18 pressure signals marked by $B$ in panel $A$ constitute two narrow bands corresponding to two different levels of HR baseline, and the oxbit moves posteriorly along the z-axis corresponding to HR acceleration and then soon moves anteriorly corresponding to subsequent deceleration. When the HR acceleration occurs during multiple heart beats (marked by $D$ in panel $A$, and panel D), the orbits moving anteriorly constitute a wide band compared with the single acceleration (panel B). The subsequent single deceleration corresponds to the orbit, which moves anteriorly.

\section{DISCUSSION}

The beat-to-beat HR; i.e., IHR, in avian embryos developing inside the eggshell is determined from cardiogenic signals detected noninvasively or invasively $(2,7,12,24)$. Some of the cardiogenic signals used for IHR determination are ballistocardiogram (BCG), acoustocardiogram (ACG), electrocardiogram (ECG) and arterial blood pressure. IHR is calculated from the time interval between the two adjacent peak waves. The ECG which has sharp spike waves seems to be the most favorable cardiogenic signal for IHR determination. In avian eggs, the ECG is detected by electrodes implanted immediately inside the shell membrane or the chorioallantoic membrane in order to minimize injury to the embryo and egg contents, However, the ECG signal is often disturbed by embryonic activities, because the electrodes do not contact directly the body of the embryo, resulting in frequent interruption of IHR determination. Compared with the ECG and other cardiogenic signals, the blood pressure which is detected by the needle catheter implanted into the allantoic artery is less influenced by embryonic activities. Höchel et al. 71 measured the blood pressure of the allantoic artery in chick embryos and presented the IHR by determining the time interval between the peaks of pressure waves (i.e., maximum blood pressure). The question that arises is, in the event that the HR is
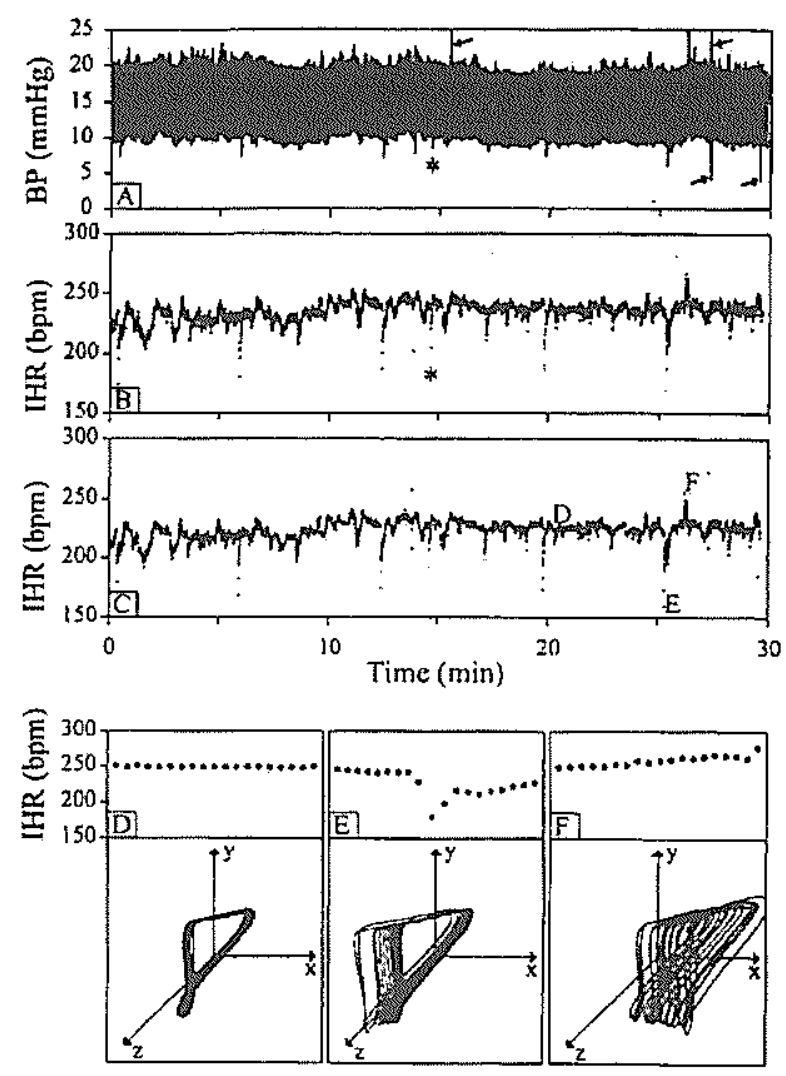

Fig. 3. The arterial pressure (BP, panel A), instantaneous heart rate (IHR) calculated from time intervals of the two adjacent peaks of blood pressure (panel $B$ ), IHR derived from the orbit reconstuetion (panel C), and reconstructed orbits (panels D, E and F) corresponding to IHR indicated by the letters, $D, E$ and $F$, respectively, in panel $C$. Time delay $(\tau)$ used for orbit reconstruction was $30 \mathrm{msec}$. Asterisk in panels $A$ and $B$ indicate the arterial blood pressure and IHR which correspond to the arterial pressure and reconstructed orbits shown in panels $A$ and $B$ of $F i g .2$. Arrows in panel $A$ are the same as in panel $\mathrm{A}$ of $\mathrm{Fig}$. I, indicating artefacts induced by embryonic activities. Other spikes and fluctuations of blood pressure in general seem to coincide chronologically with HR decelerations and accelerations.

calculated from the time interval between the minimum blood pressures or from the time interval between arbitrary levels of the pressure signals, is the same IHR obtained? The present determination of IHR from the reconstructed orbits showed that the IHR determined from the maximum blood pressure wave was the same as that derived from an arbitrary level of the pressure signals (Fig. 3). This suggests that even if the IHR is calculated from the minimum blood pressure, the same IHR is obtained and the conventional way of IHR calculation from the maximum pressure wave may be acceptable. Additionally, there is evidence that the IHR derived from the 
reconstructed orbits coincides with the IHR obtained in conventional calculation from the maximum blood pressure signal, certifying that physiological information of the arterial blood pressure is retained in the reconstructed orbits.
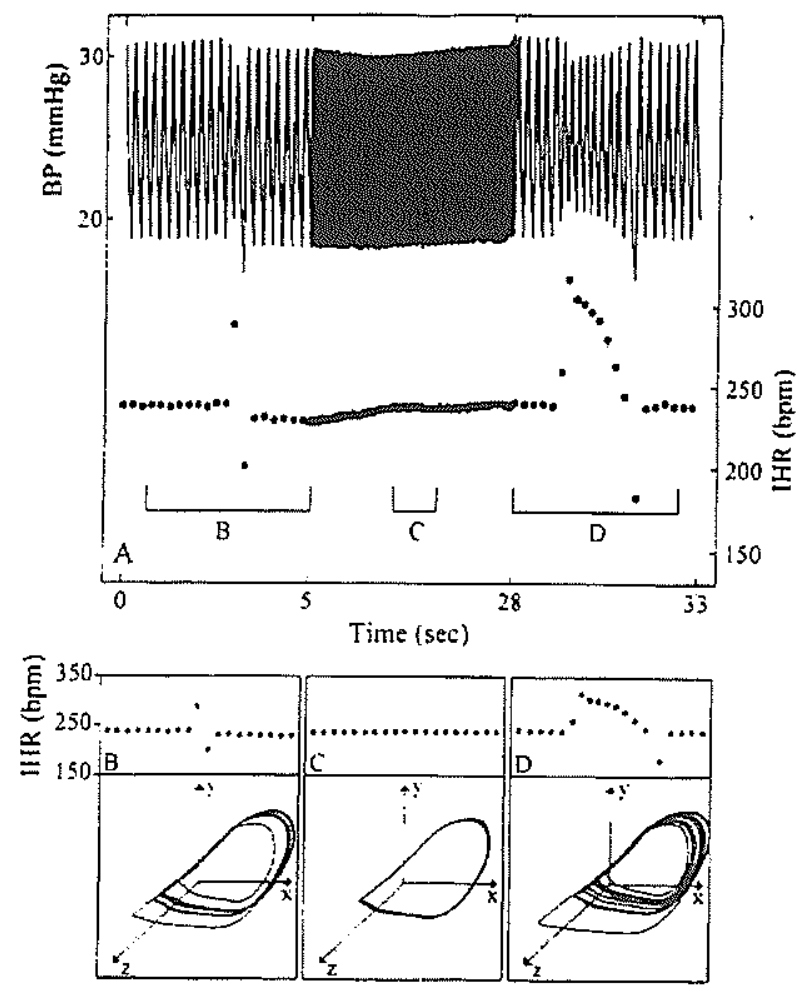

Fig. 4. The arterial blood pressure (upper tracing in panel A), IHR obtained from the pressure wave (lower tracing in parel $A$ ) and the reconstructed orbits (panels $B, C$ and D) corresponding to the arterial blood pressure indicated by the letters, $B, C$ and D, respectively, in panel A. Time delay ( $\tau$ ) was $30 \mathrm{msec}$.

In the dynamical systems approach, a time-varying single variable; i.e., the arterial blood pressure, $B P(t)$, is reconstructed by drawing of $[B P(t), B P(t+\tau)$, $B P(t+2 \tau), \cdots, B P(t+(m-1)) \tau]$. In the present report, embedding dimension, $m$, of 3 was taken to show the orbit in a visible way (i.e., in figures). $\tau$ was determined empirically to within one-tenth of a cardiac period so that the orbit could show characteristic patterns. In other words, pattern of the orbit was not fixed, but changed by the value of $\tau$. For reconstructed orbits of the blood pressures shown in Figs. 1 and 2, $\tau$ was taken arbitrarily as $40 \mathrm{msec}$ and $30 \mathrm{msec}$, respectively, to show similar orbit patterns. In the young embryo (12 days), the magnitude of the reconstructed orbits was small compared with the late embryo ( 18 days), because the pulse pressure was low. In addition, the orbits formed a narrow band centered on a closed circle in the young embryo (panel $B$ in Fig, 1) and the orbits in the 18-day-old embryo tended to move towards a direction parallel to the $x$-axis, forming the orbits as a sheet-like configuration with a wide band (panel $B$ in Fig. 2). The direction of movement and width of the band during several cardiac cycles reflect the HR fluctuations (Figs. 3 and 4 ). When the HR is stable, the orbits constitute a narrow band (panel D in Fig. 3 and panel $\mathrm{C}$ in Fig. 4). When the HR changes irregularly, the orbits form wide bands (panels $E$ and $F$ in Fig. 3 , and panels $B$ and $D$ in Fig. 4). The HR fluctuations comprising transient deceleration and acceleration are presented by orbits moving in opposing directions corresponding to decelerated HR (panel $\mathrm{E}$ in Fig. 3) and accelerated HR (panel F in Fig. 3). In other examples of orbits representing a combination of a single acceleration and deceleration (panel $B$ in Fig. 4) and sustained accelerations with single decelexation (panel D in Fig. 4), the orbits shrink along the z-axis corresponding to the HR accelerations and then widen when the single deceleration occurs. However, we do not know at present the physiological significance of these orbit patterns.

As far as the IHR is concerned, it is sufficient to determine IHR from the time intervals of the maximum blood pressure and thus record time-series data only for a part of the maximum presure signals. Meanwhile, the IHR obtained from the chick embryos shows various fluctuation patterns that intexest us as to whether they are deterministic phenomena. If we have time-series signals for the arterial blood pressure for the whole cardiac periods, the dynamical systems analysis (deterministic approach) can be attempted. In the present report, we could show characteristic figures of the reconstructed orbits for the arterial blood pressure of the embryos and derive IHR from the reconstructed orbits. Since this is the first attempt at using the dynamical systems approach, we assumed an embedding dimension, $m$, to be 3 . The next step for orbit reconstruction should be to work on a higher dimensional space ( $i . e ., m$ should be more than 3) and additionally a correlation dimension should be determined. Although in future studies additional calculations must be made to examine whether the HR fluctuations are chaotic phenomena, the HR fluctuations comprised of heart rate variability and heart rate irregularities will make favorable biological subjects for nonlinear time-series analysis.

\section{ACKNOWLEDGMENT}

This research was supported in part by a Grant-in-Aid for Scientific Research (H.T., No.08650471) of the Ministry of Education, Science and culture; the Monbusho.

\section{REFERENCES}

(1) Akiyama, R., Mitsubayashi, H., Tazawa, H. and Burggren, W. W. , Heart rate responses to 
altered ambient oxygen in early (days 3-9) chick embryos in the intact egg. J. Comp. Physiol. B 169, (1999), p85-92.

(2) Akiyama, R., Ono, H., Hóchel, J., Pearson, J. T. and Tazawa, H., Non-invasive determination of instantaneous heart rate in developing avian enbryos by means of acoustocardiogram. Med. \& Biol. Eng. \& Comput. 35, (1997), p323-327.

(3) Burggren, W.W. and Keller, B. B., (eds). Development of Cardiovascular systens: Molecules to Organisms. Cambridge Univ. Press, Cambridge, (1997).

(4) Cain, J. R., Abbott, U. K, and Rogallo, V. $L$., Heart rate of the developing chick embryo.Proc. Soc. exp. Biol. Med. 126, (1967), p507-510.

(5) Guckenheimer, I. and Holmes, P., Nonlinear Oscillations, Dynamical Systems, and Bifurcations of Vector Fields. SpringerVerlag, Heidelberg, (1983).

(6) Hashimoto, Y., Narita, T. and Tazawa, H., Cardiogenic ballistograms of chicken eggs: comparison of measurements. Med. \& Biol. Eng. \& Comput. 29, (1991), p393-397.

(7) Höchel, J., Akiyama, R., Masuko, T., Pearson, J. T., Nichelmann, M. and Tazawa, H. , Development of heart rate irregularities in chick embryos. Am. J. Physiol. 275, (1998), pH527-H533.

(8) Howe, R. S., Burggren, W. W. and Warburton, S. J., Fixed patterns of bradycardia during late embryonic development in domestic fowl with C locus mutations. Am. J. Physiol. 268, (1995), pH56-H60.

(9) Laughifn, K. F., Lundy, H. and Tait, J. A., Chick embryo heart rate during the last week of incubation: population studies. Br. Poult. Sci. 17, (1976), p293-310.

(10) Lewin, R., Dörner, M. and Tönhardt, H., Pulse oximetry: a new way of determining the heart rate in chicken embryos. Pflügers Arch - Eur. J. Physiol. 434, (1997), p639-641.

(11) Ono, H., Akiyama, R., Sakamoto, Y., Pearson, J. T. and Tazawa, H., Ballistocardiogram of avian eggs determined by an electromagnetic induction coil. Med. \& Biol. Eng. \& Comput. 35, (1997), p431-435.

(12) Pearson, J. T., Tsuzuki, M., Nakane, Y., Akiyama, R. and Tazawa, H., Development of heart rate in the precocial king quail, Coturnix chinensis. J. Expl. Biol. 201, (1998), p931-941.

(13) Pirow, R., Bilsing, R., Nichelmann, M. and Hôthel, J., A method for noninvasive, long-term recording of the avian embryo heart rate. Physiol. Behav. 58, (1995), p185-189.

(14) Rahn, H., Poturalski, S. A. and Paganelli, C. V., The acoustocardiogram: a noninvasive method for measuring heart rate of avian embryos in ovo. J.Appl. Physiol. 69, (1990), p $1546-1548$.

(15) Sakamoto, Y., Haque, M. A., Ono, H., Pearson, J. and Tazawa, H., Two-dimensional cardiogenic ballistic movements of avian eggs. Med. Biol. Eng. \& Comput. 33, (1995), p611-614.

(16) Suzuki, Y., Musashi, H. and Tazawa, H. Noninvasive heart rate monitoring system for avian embryos based on ballistocardiogram. Med. \& Biol. Eng. \& Comput. 27, (1989), p399-404.

(17) Takens, F., Detecting Strange Attractors in Turbulence. Lecture Notes in Mathematics, 898, Springer-Verlag, Heidelberg, (1981), p366-381.

(18) Tazawa, H. , Measurement of blood pressure of chick embryo with an implanted needle catheter. J. Appl. Physiol. 51, (1981), p1023-1026.

(19) Tazawa, H., Ar, A., Rahn, H. and Piiper, J., Repetitive and simultaneous sampling from the air cell and blood vessels in the chick embryo. Respir. Physiol. 39, (1980), p265-272.

(20) Tazawa, H., Hashimoto, Y. and Doi, K., Blood pressure and heart rate of chick embryo (Gallus domesticus) within the egg: responses to autonomic drugs. In: R.B. Hill, K. Kuwasawa, B. R. McMahon and T. Kuramoto (eds.) Phylogenetic models in functional coupling of the cNs and the cardiovascular system. Karger, Basel, (1992), p86-96.

(21) Tazawa, H., Hashimoto, Y., Takani, M., Yufu, Y. and Whittow, G. C., Simple, noninvasive system for measuring the heart rate of avian embryos and hatchlings by means of a piezoelectric film. Med. \& Biol. Eng. \& Comput. 31, (1993), p129-134.

(22) Tazawa, H., Hiraguchi, T., Asakura, T., Fujii, H. and whittow, G. C., Noncontact measurements of avian embryo heart rate by means of the laser speckle: Comparison with contact measurements. Med. \& Biol. Eng. \& Comput. 27, (1989), p580-586.

(23) Tazawa, H. and Hou, P-C. I., Avian cardiovascular development. In: Burggren, W. W. and B. B. Keller (eds) Development of Cardiovascular systems: Molecules to Organisms. Cambridge Univ. Press, Cambridge, (1997), p193-210.

(24) Tazawa, H., Mitsubayashi, H., Hirata, M. Höchel, J. and Pearson, J. T., Cardiac rhythms in chick embryos during hatching. Comp. Biochen. Physiol. (in press, also this volume), (1999).

(25) Tazawa, H. and Nakagawa, S., Response of egg temperature, heart rate and blood pressure in the chick embryo to hypothermal stress. J. Comp. Physiol. B 155, (1985), p195-200.

(26) Tazawa, H., Suzuki, Y. and Musashi, H., Simultaneous acquisition of ECG, BCG and blood pressure from chick embryos in the egg. J. Appl. Physiol. 67, (1989), p478-483. 


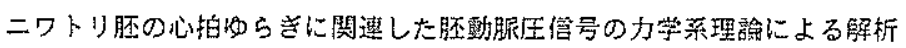

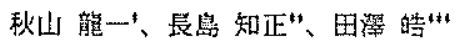

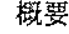

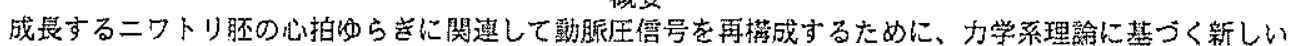
解析を訊对た。この解析法は、単一の暗系列データによって衰されるーつの現象をモデル化し、任意のシス

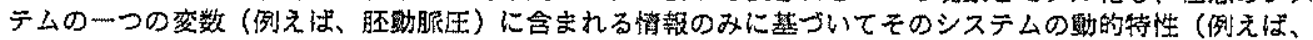

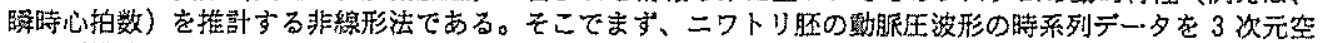

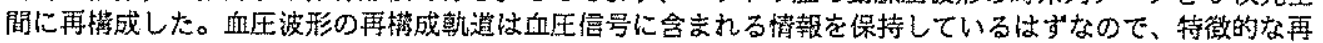

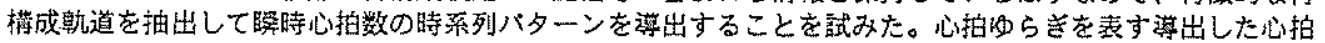

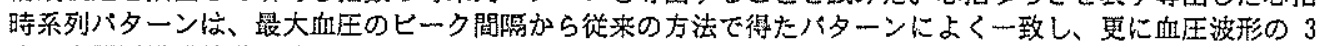

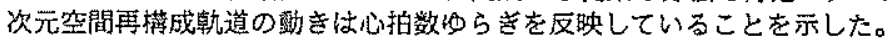

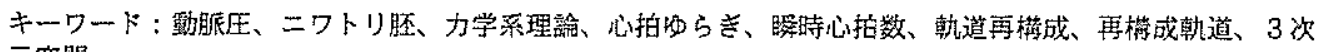
实然間

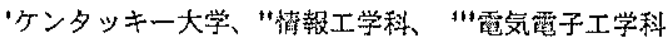

